\title{
Location Based Logistics Services and Event Driven Business Process Management
}

\author{
Christoph Emmersberger, Florian Springer, and Christian Wolff \\ Senacor Technologies AG, Vordere Cramergasse 11, 90478 Nürnberg, Germany \\ \{christoph.emmersberger, florian.springer\} @senacor.com \\ University of Regensburg, 93040 Regensburg, Germany \\ christian.wolffacomputer.org
}

\begin{abstract}
Location-based Services (LBS) [1] have already started their market penetration process and several platforms capable of running LBS are available. With the number of LBS increasing, the current development is targeting consumer applications, although LBS have a high potential for enhancing business processes in companies as well. Considering business process optimization, one concept recently discussed in the field of Business Process Management (BPM) [2] is Event-Driven Business Process Management (ED-BPM) [3], which combines Business Process Management (BPM) and Complex Event Processing (CEP) [4]. This paper introduces ED-BPM for LBS in the logistics field, exemplifying its potential use with an example for a logistics order process execution.
\end{abstract}

Keywords: ED-BPM - Event-Driven Business Process Management, LBS Location-based Services, CEP - Complex Event Processing, GPS - Global Positioning System.

\section{Introduction}

This section provides an introduction to the concepts of Location-based Services (LBS) and Event-Driven Business Process Management (ED-BPM) in the context of logistics. It also gives an overview of the current market situation in the logistics industry sector. J. Schiller and A. Voisard define Location-based Services as "a recent concept that denotes application integrating geographic location (i.e. spatial coordinates) with the general notion of services" [1, page 1]. This means that LBS offer information related to their current location over a network, usually by using mobile devices. The fundamentals of LBS are based on military location systems (e.g. the Global Positioning System (GPS)) and mobile network communications. A wealth of applications that offer information related to the service users' actual position has been developed so far, e. g. restaurants and sights recommendation systems [5] or applications showing which friend is close to the user [1]). Compared to existing positioning systems (e.g. mobile navigation systems with deployed maps), the information is not statically installed on the mobile device but can be updated during the application execution upon a service request. Looking beyond consumer services, LBS obviously have a high potential for enhancing business processes as well. In this 
paper we identify several services which could enhance current logistics order handling processes with more flexibility.

"The term Event-Driven Business Process Management is a combination of actually two different disciplines: Business Process Management (BPM) and Complex Event Processing (CEP)."[3]. BPM is seen as a management discipline to "support the design, enactment, management and analysis of operational business processes." [6]. This means that the business processes express each company's unique way of running their business which is their most valuable asset [3]. Processes are triggered by events; at the same time, they may publish events themselves during process execution. An event can be described as "anything that happens or is contemplated as happening" [7, page 5]. Events are sent over event processing networks (EPN) [7] where they can be filtered, routed, aggregated and may result in actions. These tasks are executed by middleware components with event processing capabilities (especially CEP engines).

The rest of this paper is organized as follows: In ch. 2 we give an introduction of current challenges for information processing in the logistics domain. Ch. 3 describes a common logistics process model and gives an in-detail description of selected processes, while ch. 4. presents an analysis of both, the processes' weaknesses as well as potential for optimization by introducing innovative LBS. Finally, ch. 5 discusses how location based services may be coupled with event-driven business process management.

\section{Basic Characteristics of Logistics Companies}

Basic concepts of current logistics have been established in the second half of the $20^{\text {th }}$ century, although there is still no generally agreed-upon definition for "logistics" yet. In the literature the common understanding of logistics reaches from "simple" operations for transporting, handling, and warehousing of goods to more intricate management functions (supply chain management [8]): "Even when taking all the different opinions into account, a consensus exists [...] that the central function of logistics is the bridging of space-time disparities concerning goods and materials." [8, page 10]. To realize efficient goods and material logistics, an adequate information supply helps to provide a "(...) more precise picture of the sales development" [9] as well as to face the current market situation.

In this paper we highlight several LBS examples based on current logistics order processes for small and large logistics service providers (large package deliverers as well as smaller courier service providers). According to the Messenger Courier Association of America (MCAA), the difference in the business models is that the so called "big four" "DHL, UPS, FedEx and USPS [...]" of "[...] the delivery business [...] do not provide same-day delivery services uniquely designed to meet specific individual customer needs." [10, page 3]. The reason for that can be found in the order and shipment execution processes which have been originally designed for large throughputs. Fig. 1 shows the differences of large and small companies which becomes obvious when the delivery area covered, the number of employees, or the technical infrastructure typically used are considered. 


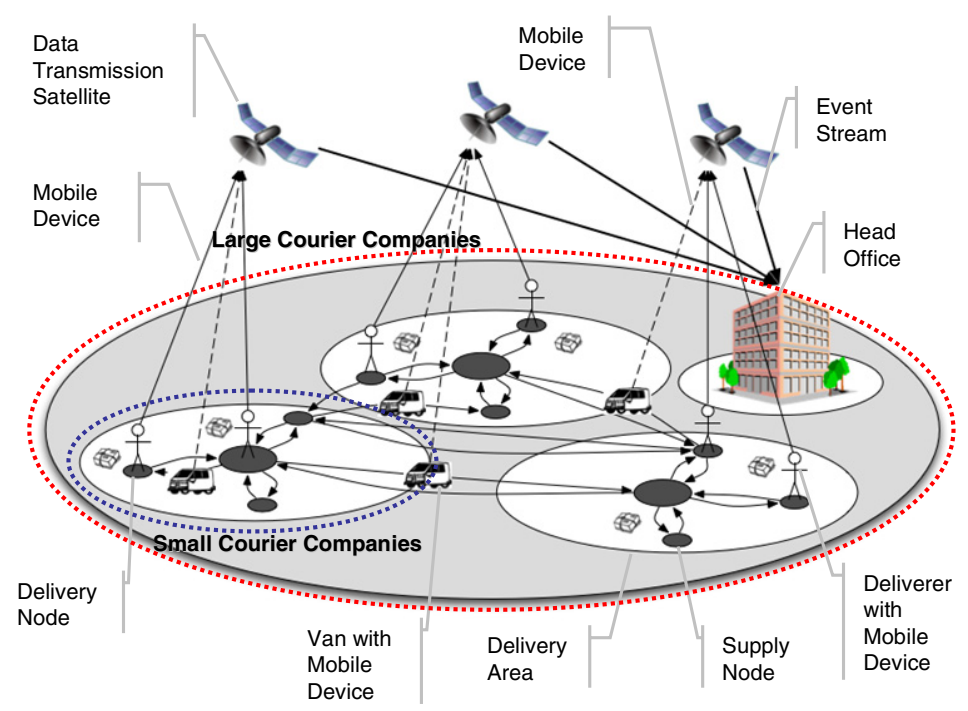

Fig. 1. Structural Differences of Small and Large Logistics Service Providers

Large logistics companies can be characterized by owning a global delivery network which is segmented into smaller pieces (i.e. regional distribution networks or delivery areas (Fig. 1)) to obtain the manageability of logistics complexity. The physical delivery process is not executed as a direct relation from the supply to the delivery node, and contains usually several steps over different distribution nodes.

Large companies already use several types of mobile devices (e.g. navigation systems or handheld scanners) to keep track of the state of their delivery processes. They operate on day-based, fixed-calculation production plans which can not be adjusted during their execution. Their business is optimized for delivering huge quantities of goods in small timeframes. The global quantity of delivered items is typically greater than 1 million items per day [14].

Small logistic companies or courier services work in small delivery areas like a city or a community instead of operating on a global delivery network. Therefore these companies usually can only offer their services within their own delivery area with the exception of cooperation with other service providers working in different delivery areas (Fig. 1). In comparison with big logistics companies, typically a single person is responsible for the whole delivery process from the delivery to the supply node including pick up, transport and delivery of packages. Small courier companies have to deliver their goods based on legal stipulations [15]. A stipulation is the traceability within the delivery process. Therefore several steps of the process have to be logged or journalized. While big logistics companies use hand scanners for this step, courier services often perform this manually and paper-based. In contrast to large logistics companies, small couriers deliver only a few hundred or thousands goods per day. They can act more flexibly, due to their size of typically about 25 employees [10]. In addition, the small amount of orders and the limited delivery area enhance flexibility and customer satisfaction for courier services. 
Fig. 2 identifies four main parties that put pressure on the logistics domain. The first party is determined as customer's perspective: The increasing demand for timedefinite [11] and end-to-end documented processes forces logistics companies to create faster, more flexible processes. Besides the demand for new products, customers own a huge bargaining power that results in decreasing margins.

The second force that puts pressure on the market can be identified as the threat of new competitors that create substitution products. A new competitor for the logistics industry is the IT industry that offers new collaboration services for information and document exchange which were formerly transported by logistics companies.

The third pressure party is closely connected with the new competitors, because they create a product substitution perspective: New products go beyond collaboration services mentioned above. Such services may provide online real-time information, which was formerly sold by the logistics companies.

The last party that puts pressure on the market is given by logistics suppliers which gain more and more influence, because the tendency of sourcing services to other companies encourages these companies to create more and new services. At the same time, it raises their bargaining power. This analysis should make clear that both, small as well as large companies need to improve their processes.

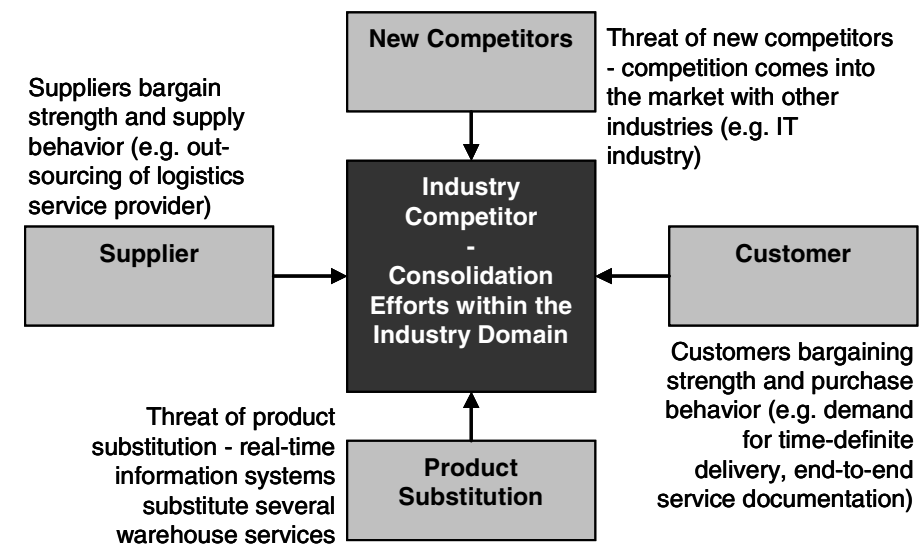

Fig. 2. Competitive Environment in the Logistics Domain derived from [11]

To exemplify the issues of small and large logistics service providers in more detail, the following section introduces state of the art logistics processes starting from a general logistics process model.

\section{The Common Logistics Process in Large and Small Companies}

Fig. 3 shows the simplified structure of a common logistics process model. The model can be applied to large and small logistics companies alike as well as to specialized customer-service providers. Similar process models can be found in [12] and [13]. 


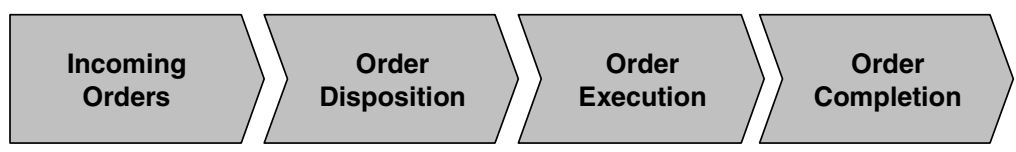

Fig. 3. Common Logistics Process Model

Based on the experience of the authors in several projects in the logistics domain, there is a large difference in executing the common logistics shown above process model depending on the business model. Based on the common model, the following section provides a more detailed process description for large and small logistics service providers. While it is idealized to a certain degree, we intend to explain the basic characteristics of current processes in logistics.

\subsection{Process Description for Large Logistics Companies}

1. Incoming Orders: Customers place their orders via e.g. internet portals, installed software, call centres or email. After the orders have been placed, package deliverers calculate their production plans based on the present order amounts and generate their work schedules (i.e. pickup, transportation and delivery). All items which have not been placed until a fixed time (e.g. $8 \mathrm{pm}$ ) cannot be included in the task schedule.

2. Order Disposition: A logistics delivery process takes place between different nodes in the global network. There may be several nodes (hubs and distribution centres) between delivery endpoints that handle the routing of goods. Calculating work schedules is a complex task which be do not discuss here.

After finishing the work schedule calculation the plans are distributed over the companies' local area network (LAN) to the mobile devices. The reason for the data transmission via LAN is the widespread delivery area with a partially missing area-wide mobile high speed network connection (e.g. UMTS) enabling the transaction of a large amount of data. After the initial load is finished, the deliverer can start with order execution.

3. Order Execution: During order execution, the deliverer approaches several nodes to pickup packages which have been pre-announced from customers by their order placement. At each node, packages labels are scanned to record their current order state. Besides the tracking of the order process, the scan is also used to confirm the transfer of risks and liabilities.

During transportation, there can be several transit nodes where different distribution mechanisms occur (e.g. truck trailer exchange, manually sorting mechanisms, machine-based sorting). At these transfer nodes the packages need to be scanned again to throw state-change enabling tracking events. While the process is executed, a huge amount of events is collected from each single transported item.

4. Order Completion: At the package delivery node, the recipient confirms the successful transfer of perils by signing the order fulfilment form e.g. on a handheld scanner. The confirmation generates a final tracking event which is sent to the central information system. The order will be marked as successfully completed in the IT system and will be available for further processing in a financial services system, for example. 


\subsection{Process Description for Small Logistics Companies}

1. Incoming Order: Currently, customers place there order via telephone, fax or email. The orders are directly placed in the company's head office or call center. An order for a courier service can be a pick up of an important compact disc (CD) with the layout of a flyer which has to be delivered to the printer or the transportation of a transplant from one hospital to another. Customers also place continuous orders where a courier has to pickup and deliver the daily post from the client.

2. Order Disposition: Depending on the priority of the order, staff decides manually and in many cases without software support on the point in time when the order has to be executed. High priority orders (express courier service) will be directly transmitted via cell phone to the responsible staff member. Orders with a lower priority (standard courier service) are collected and transmitted to the service staff on the next day or in a free time slot.

3. Order Execution: Before start of work the service staff gets a list of tasks to be executed during the working day. The execution of the schedules is sequentially except for high priority orders.

4. Order Completion: After a single order is completed, service staff starts with the tasks of the next order. Usually, service staff completes all assigned orders before the head office is informed about the order status.

\section{Process Analysis: Weaknesses and Potentials}

The difficult market situation forces logistics companies to optimize their business processes [16]. Based on the common process model, the main issues for large as well as small logistics companies are identified (Fig. 4).

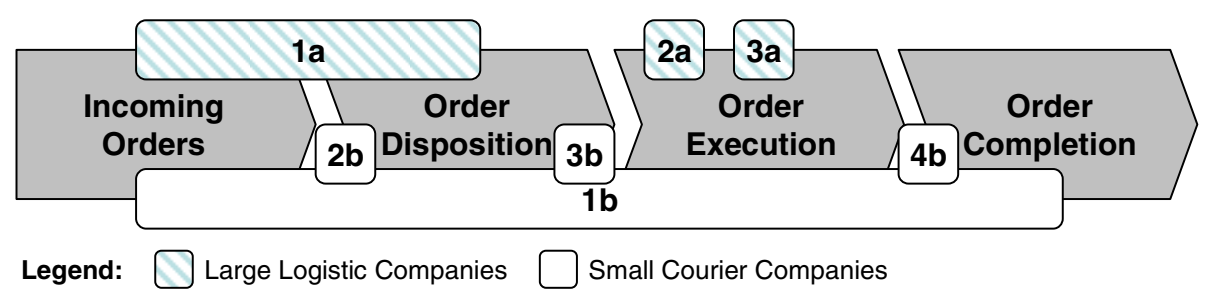

Fig. 4. Process Issues of Large and Small Logistics Companies

\subsection{Process Issues of Large Logistics Companies}

1a. Incoming orders that arrive after the deadline for order disposition cannot be considered for the next work schedule, even if the capacity utilization is not even close to $100 \%$.

2a. Once the route schedules are calculated and pushed on the navigation systems, they are static and can not be adjusted according to current traffic and order situation. 
3a. Due to the large amount of tracking events and the known limitations of mobile network connections, the actual state of the delivery process is an ex-post calculation. Adjustments based on past experience might mess up the ongoing delivery process. Enhancements in information supply can increase the flexibility for order disposition as well as enhance the resource efficiency.

\subsection{Process Issues of Small Logistics Companies}

1b. Due to manual (e.g. status report via cell phone) or paper-based journalizing approaches a real-time introspection of the order status is not possible. Automatically and electronically transmitted status responses, e.g. via special mobile devices have the potential to enable real-time insight and reduce the effort of the staff during the process. Insight about orders is also necessary to assure optimal order disposition.

2b. Currently, time- and cost-efficient order disposition is not possible: An overview of all orders and their respective status as well as the geographical position of service staff is needed to dispose tasks in an optimal way. This is primarily important for incoming, high priority orders which have to be executed immediately. The service staff has to be equipped with a mobile device that allows sending the geographical position as well as the order state.

3b. Assignment of orders from the head office to the service staff is currently done manually via cell phone calls. Modern mobile devices allow transmission of orders directly to the mobile device of the service staff.

4b. Currently, service staff has to take care of reaching the right destination in an efficient way. The reason for that is that the staff has no information about the actual traffic situation, etc. The taken route is in most cases not the optimal track. Modern navigation systems help resolving this issue.

Table 1. Location Based Services for Process Enhancement

\begin{tabular}{|l|l|l|}
\hline & $\begin{array}{l}\text { Courier Service } \\
\text { Provider }\end{array}$ & $\begin{array}{l}\text { Large Package } \\
\text { Deliverer }\end{array}$ \\
\hline $\begin{array}{l}\text { Optimal Order Disposition based on } \\
\text { Order State and Geographical Position }\end{array}$ & ++ & + \\
\hline $\begin{array}{l}\text { Electronically order Transmission } \\
\text { depending on Geographical Position of } \\
\text { the Courier }\end{array}$ & ++ & $+\mid 0$ \\
\hline $\begin{array}{l}\text { Rerouting of goods depending on the } \\
\text { geographical position of the receiver }\end{array}$ & - & ++ \\
\hline $\begin{array}{l}\text { Effective Route Scheduling and Guidance } \\
\text { by Using Different Event Sources }\end{array}$ & + & + \\
\hline $\begin{array}{l}\text { Potential process improvement and } \\
\text { relevance: }\end{array}$ & $\begin{array}{l}++ \text { very high } \\
+ \text { high }\end{array}$ & $\begin{array}{l}0: \text { low } \\
- \text { not relevant }\end{array}$ \\
\hline
\end{tabular}

\subsection{Location Based Services for Process Enhancements}

Based on the process issues identified above several LBS can be identified to improve the logistics companies' processes. Tab. 1 gives an overview of such LBS along with 
their relevance for small and large logistics companies. The suggestions will be discussed in more detail below.

\subsubsection{Optimal Order Disposition Based on Order State and Geographical Position}

To resolve one of the existing issues of the order execution process, a LBS for optimal order disposition based on real-time order-state confirmation was identified. Status information combined with the geographical position of the courier or service employee enables the logistics company to react on short term, high priority orders in the way of re-scheduling the order disposition. The re-scheduling occurs e.g. when a customer calls in, to place an order for an express shipping. The disposition of this order can be calculated based on the closest distance of a service employee to the customer. The second restriction which has to be considered for re-scheduling the order disposition is the capacity of the courier. These can be calculated based on the order-state information sent through mobile devices. Optimal order disposition has to be done centrally in the head office based on the transmitted status information of the couriers.

\subsubsection{Electronically Order Transmission Depending on Staff Position}

In order to enable an optimal order disposition, a mobile device needs the functionality of receiving the disposed data. The disposition has to be calculated based on the current position of the courier and needs to be published to the mobile device to provide the new information containing the changed working schedule.

In large companies this mechanism is currently not possible because of the non-availability of real time status data and missing area-wide network connections, in small companies it is executed manually via calls on the mobile phones. Recently released mobile devices and the growing network accessibility create the potential to extend the existing functionalities for enabling an automatic order transmission. This extension creates more efficiency by reducing manual task and enabling more process flexibility.

\subsubsection{Rerouting of Goods Depending on the Geographical Position of the Receiver}

The increasing mobility of people creates the customers' demand for receiving their packages at their current whereabouts. An extreme example for the increasing mobility is the life of business consultants who are usually working during the week at their customer's site and not in their place of residence. If they urgently need media (e.g. books, newspapers, magazines) they had ordered before they left, the order process needs to be adjustable to sending the goods to the place where they stay. That demand requires short term re-routing which can be realized as follows: After a package has been picked up at the delivery node, the receiver retrieves information via SMS or email about the beginning of the shipment. After the information has been delivered, the receiver can decide to which place he wants to get the package. If he likes to change the delivery address he needs to response to the SMS or email with an updated delivery address. 


\subsubsection{Effective Route Scheduling and Guidance by Using Different Event Sources}

The inclusion of different event sources enables a more efficient delivery process: Such event sources may include a traffic message channel (TMC) which transmits current traffic information. The information can be used by correlating these events with the scheduled routes of the transportation vehicles to enable re-routing in case of traffic congestions. Other event sources in that context are e.g. weather, flight and train information to enable forecasting as well as avoiding delivery delays. A proof of concept that evaluates technologies for achieving this approach already exists [17].

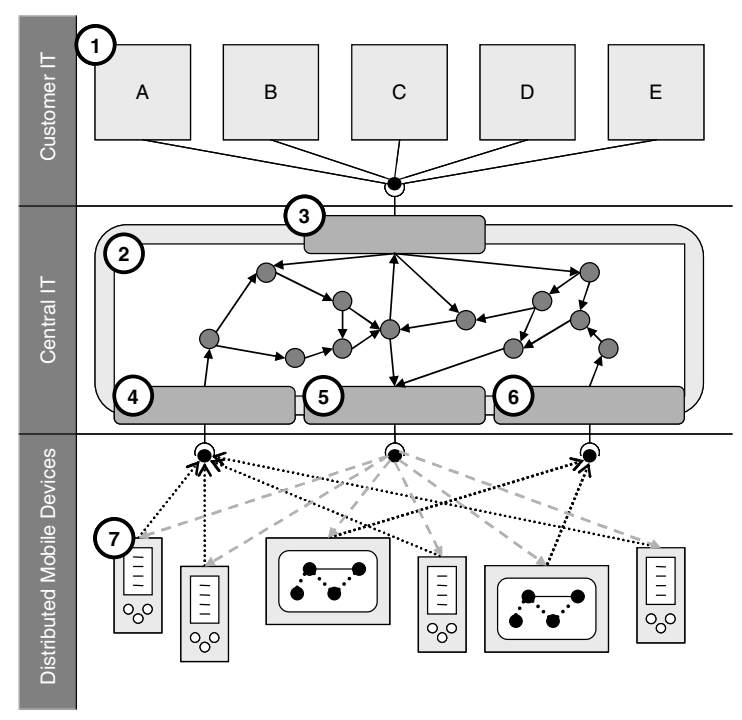

Fig. 5. Architectural Overview for a Combined LBS - ED-BPM Solution

\section{Combining LBS with Event Driven Business Process Management}

For realizing the proposed LBS that are designed to resolve the current issues in the logistics domain, the solution approach is comparable to existing ED-BPM reference models for other industry domains, like for example in the financial services domain [18]. Taking a look at the logistics context, the conceptual architecture has three layers (Fig. 5): The "Customer IT" layer, onto which the logistics company has limited influence only, the "Central IT" layer of the logistics company which hosts all applications that affect the logistics service provider and the "Distributed Mobile Devices" layer which is spread out over the delivery areas. The following enumeration explains the solution architecture of Fig. 5 based on the numbers posted in the overview diagram:

1. Customers IT Systems: Each customer has its own IT systems, which are realized on different technologies from different software vendors. These application systems need to be connected to the central IT systems of the logistics service provider to enable order placement and state-information reception. 
2. Event Correlation Engine: The central component of the logistics companies' IT infrastructure is an event correlation engine or complex event processing engine. This component correlates information coming from customers, the mobile devices in the delivery areas and from potential other sources (e.g. traffic- and weather information) to provide a real-time insight in the ongoing processes. The correlations and aggregations are deposited as event patterns. If an event pattern matches, the engine is capable to send a new, complex event to the affected peripheral systems. The communication relation the surrounding peripheral systems is realized through so called event adapter.

3. Event Adapter for Customers IT Systems: The event adapter for customer IT systems is responsible for retrieving orders as well as sending state information to the customers. It is a logical adapter and is implemented on several technologies, depending on the deployed applications.

4. Event Adapter for Receiving Order State Information: The event adapter for receiving order state information is placed at the border between the central IT system and the distributed mobile devices. The adapter collects all information from the mobile devices related to the current state of an order.

5. Event Adapter for Position Information: The adapter for position information gathers the location information of the mobile devices. When using the global positioning system, the information is declared as longitude and latitude. Other possible position information can be derived from mobile transmission towers.

6. Event Adapter for Distribution of Re-Scheduled Working Tasks: If an event pattern that causes a re-scheduling of current tasks has matched in the CEP engine, the information needs to be published through a specific event adapter. This means that changes in existing route and working schedules are published to the mobile devices while they operate in the delivery areas resulting in dynamic real time adaptation of routes and schedules.

7. Mobile Devices: Mobile devices are used to support the order execution process. They route the deliverers to their next destinations and keep them informed about the orders they have to accomplish. Mobile devices enable the tracking of the current order state, the actual position of the packages and the employee's position for extending the existing order disposition and routing processes. To achieve this enhancement their functionality is extended by LBS as well as EDBPM in combination with adequate data loading and distribution mechanisms.

\section{Conclusion}

This paper provides analyses current issues in process management in the logistics domain and discusses the coupling of location based services and event-driven business process management. Following the identification of current issues in the delivery process of large and small logistics service providers, we suggest four location based services for order scheduling and route planning and propose an architectural sketch for LBS / ED-BPM in logistics. While there are still major conceptual as well as technical problems that need to be resolved (e. g. area-wide mobile network coverage), the concept sketched above helps to understand how LBS may be applied in the daily business of a logistics company. The core issue of modeling and detecting relevant complex events in this model will be a key issue for further research in this area. 


\section{References}

[1] Schiller, J., Voisard, A.: Location-Based Services. Elsevier the Morgan Kaufmann Series (May 14, 2004) ISBN: 1558609296

[2] Chang, J.F.: Business process management systems: strategy and implementation. CRC Press, Boca Raton (2006)

[3] Özsu, M.T., Liu, L.: Encyclopedia of Database Systems, 1st edn. Springer, Heidelberg (2009)

[4] Luckham, D.: The Power of Events: An Introduction to Complex Event Processing in Distributed Enterprise Systems. Addison-Wesley Professional, Reading (2002)

[5] Focus online Digital: Umgebung checken: Around me, http://www. focus.de/ digital/handy/iphone/tid-14047/iphone-apps-umgebung-checkenaround-me_aid_394119.html (Downloaded 2009/06/08)

[6] Aalst, W.v., Hofstede, A.t., Weske, M.: Business Process Management. In: van der Aalst, W.M.P., ter Hofstede, A.H.M., Weske, M. (eds.) BPM 2003. LNCS, vol. 2678, pp. 1-12. Springer, Heidelberg (2003)

[7] Luckham, D., Schulte, R.: Event Processing Glossary - Version 1.1. Event Processing Technical Society (July 2008)

[8] Deepen, J.M.: Logistics Outsourcing Relationships. Physica-Verlag, Heidelberg (2007)

[9] Dyckhoff, H., Lackes, R., Reese, J.: Supply chain management and reverse logistics. Springer, Heidelberg (2003)

[10] MCAA Messenger Courier Association of the Americas, Same-Day Delivery and the Messenger Courier Industry: A Profile of the Less than 24 Hour Delivery Industry and its Value, Washington DC (2006)

[11] Pfohl, H.-C., Dalquen, P., Deller, M., et al.: Güterverkehr - eine Integrationsaufgabe für die Logistik. Erich Schmidt Verlag GmbH (2003) ISBN 9783503074587

[12] Ordys, A.W., Uduehi, D., Johnson, M.A., Thornhill, N.F.: Process control performance assessment: from theory to implementation. Springer, Heidelberg (2007)

[13] Windt, K., Hülsmann, M.: Understanding Autonomous Cooperation and Control in Logistics: The Impact of Autonomy on Management, Information, Communication and Material Flow. Springer, Heidelberg (2007)

[14] Goldschmitt, W.: DHL Express Post beendet US-Abenteuer, Frankfurter Rundschau, (November 11, 2008),

http: //www.fr-online.de/in_und_ausland/wirtschaft/aktuell/ 1627600_Post-beendet-US-Abenteuer.html (Downloaded 2009/06/12)

[15] Regulierungsbehörde für Telekommunikation und Post (20.04.2002), http://www . kurier.com/sites/bdkep/doku/2df_offermann-Thesen.pdf

[16] BdKEP - Bundesverband der Kurier-Express-Post-Dienste e.V., KEP aktuell - Dem KEPMittelstand gebührt Aufmerksamkeit (January 2009), http: / /www.kurier.com/2007-04-14/artikel/15-2009.pdf

[17] Springer, F., Emmersberger, C.: Event Driven Business Process Management taking the Example of Deutsche Post AG - An evaluation of the Approach of Oracle and the SOPERA OpenSource SOA Framework. University of Applied Sciences, Diplomarbeit (2008), http: / /www.citt-online.de/downloads / EmmSpr_Diplomarbeit_Final.pdf

[18] Ammon, R. v., Emmersberger, Ch., Ertlmaier, Th., Etzion, O., Paulus, Th., Springer, Fl.: Existing and Future Standards for Event-Driven Business Process Management. In: Proc. DEBS, Third Int'l Conference on Distributed Event-Based Systems, Nashville/TN (to appear, 2009) 
